\title{
Effects of Age on Cerebrospinal Fluid Oxytocin Levels in Free-Ranging Adult Female and Infant Rhesus Macaques
}

\author{
Karen J. Parker \\ Stanford University School of Medicine \\ Shellie A. Hyde and Carl S. Cummings \\ Stanford University School of Medicine
}

Christy L. Hoffman

University of Chicago

\author{
Dario Maestripieri \\ University of Chicago
}

\begin{abstract}
There is growing interest in examining oxytocin and social functioning in human and non-human primates. Studies of human oxytocin biology are typically restricted to peripheral assessments because opportunities to collect cerebrospinal fluid (CSF) are rare. Several studies have examined CSF oxytocin levels in captive adult primates, but none to our knowledge have been conducted under free-ranging conditions and inclusive of infants. The main goal of this study was to establish feasibility of quantifying CSF oxytocin levels in free-ranging adult female and infant rhesus monkeys living on Cayo Santiago, PR. CSF oxytocin levels were examined in relation to individuals' demographic and reproductive characteristics as well as plasma cortisol levels. CSF oxytocin concentrations ranged from 36.02 to 134.41 $\mathrm{pg} / \mathrm{ml}$ in adult females (ages 7-26 years; $N=31$ ) and 35.94 to $77.3 \mathrm{pg} / \mathrm{ml}$ in infants (ages 38-134 days; $N=17$ ). CSF oxytocin levels were positively correlated with adult female age and negatively correlated with infant age. The former correlation was driven by reproductive status. CSF oxytocin levels were unrelated to dominance rank or plasma cortisol levels. In contrast to a previous study of plasma oxytocin concentrations in this population, CSF oxytocin levels did not differ significantly between lactating and non-lactating females. These findings: 1) provide feasibility data for examining CSF oxytocin levels in free-ranging non-human primates and 2) indicate that CSF oxytocin levels may be a biomarker of age-related central nervous system changes across lifespan development. Research is now required to examine CSF oxytocin levels in the context of social functioning in free-ranging rhesus monkeys.
\end{abstract}

Keywords: age, cerebrospinal fluid, infant, cortisol, free-ranging, oxytocin, rhesus monkey

Oxytocin is a nine-amino acid peptide hormone which is synthesized in the hypothalamus and released into systemic circulation via the posterior pituitary. Oxytocin is also released into the central nervous system via widely distributed oxytocinergic pathways (Gimpl \& Fahrenholz, 2001). There is growing interest in studying oxytocin biology in human and nonhuman primates because of strong evidence from rodent studies demonstrating the critical role of central oxytocin in regulating a variety of social behaviors (Carter, Grippo, Pournajafi-Nazarloo, Ruscio, \& Porges, 2008; Lim \& Young, 2006; Pedersen, 2004). Studies of humans
Karen J. Parker, Shellie A. Hyde, and Carl S. Cummings, Department of Psychiatry and Behavioral Sciences, Stanford University School of Medicine; Christy L. Hoffman and Dario Maestripieri, Department of Comparative Human Development, University of Chicago.

Funding for this study was provided by NIH Grant R21-AG029862 to D.M. and by a NARSAD Young Investigator Award to K.J.P. NIH and NARSAD had no further role in study design; in the collection, analysis and interpretation of data; in the writing of the report; and in the decision to submit the paper for publication. This publication was made possible by grant number CM-5P40RR003640 from the NIH National Center for Research Resources (NCRR) to the Caribbean Primate Research Center of the University of Puerto Rico. Its contents are solely the responsibility of the authors and do not necessarily represent the official views of the NCRR or NIH.

We thank Richelle Fulks for assistance with sample collection, and Adaris Mas-Rivera, James Ayala, and the staff of the Caribbean Primate Research Center for logistical support and assistance with animal capture and handling. This study was conducted in accordance with the NIH Guide for the Care and Use of Laboratory Animals. The protocol for this study was approved by the Institutional Animal Care and Use Committee, Medical Sciences Department, University of Puerto Rico.
All authors state that they have no actual or potential conflicts of interest including any financial, personal or other relationships with other people or organizations within three (3) years of beginning the work submitted that could inappropriately influence, or be perceived to influence, their work.

Christy L. Hoffman and Dario Maestripieri collected the biological samples and demographic data. Dario Maestripieri coordinated with the Biomarker Assay Core Lab to have plasma cortisol levels quantified. Karen J. Parker oversaw the quantification of CSF oxytocin levels in her laboratory. Carl S. Cummings and Shellie A. Hyde developed and conducted the CSF oxytocin assays. All authors contributed to data entry. Karen J. Parker, Christy L. Hoffman, and Dario Maestripieri conducted the statistical analyses. Karen J. Parker and Dario Maestripieri wrote the manuscript. All authors contributed to and have approved the final manuscript.

Correspondence concerning this article should be addressed to Karen J. Parker, Ph.D., Department of Psychiatry and Behavioral Sciences, 1201 Welch Road, MSLS Room P104 - Mail Code 5485, Stanford University School of Medicine, Stanford, CA 94305-5485. E-mail: kjparker@ stanford.edu 
are typically restricted to peripheral oxytocin assessments due to the invasiveness of cerebrospinal fluid (CSF) collection procedures (Carter, 2007; Modahl et al., 1998; Parker et al. in press). Whether plasma oxytocin levels serve as a meaningful index of central activation is unclear, however, because central and peripheral sources of oxytocin are thought to be functionally distinct, and once released into circulation, oxytocin does not reenter the brain (Amico, Challinor, \& Cameron, 1990; Landgraf \& Neumann, 2004).

Opportunities to collect CSF are more common in monkeys than in humans, allowing more direct examination of central oxytocin biology (Robinson \& Coombes, 1993). Moreover, in monkeys, CSF can be collected from the cisterna magna, a ventricle at the base of the skull, which prevents the disappearance of peptide signal typically associated with the more distal lumbar sampling procedures utilized in humans. A few studies have measured CSF oxytocin levels in laboratory primates (Amico et al., 1990; Rosenblum et al., 2002; Winslow, Noble, Lyons, Sterk, \& Insel, 2003), but none to our knowledge have been conducted under freeranging conditions. Laboratory primates are often characterized by abnormal developmental histories and rarely live in species-typical social environments. Free-ranging primates therefore represent a more ecologically valid model by which to investigate the relationships between oxytocin biology and complex social processes.

The goal of the present study was to establish the feasibility of quantifying CSF oxytocin levels in free-ranging rhesus monkeys living on the island of Cayo Santiago. Free-ranging subjects were captured during the annual trapping period, which provided an opportunity to collect biological samples for this feasibility study. Study subjects included lactating and nonlactating adult females and infants as young as 1 month of age. CSF oxytocin levels were examined in relationship to individuals' demographic and reproductive characteristics as well as in relation to plasma cortisol levels.

\section{Method}

\section{Subjects}

This study was conducted with the free-ranging population of rhesus macaques on Cayo Santiago, a 15.2 ha island located $1 \mathrm{~km}$ off the southeastern coast of Puerto Rico. During the study period, the population included approximately 850 animals distributed among six naturally formed social groups. Monkeys on Cayo Santiago forage on vegetation and are provisioned with rainwater and commercial monkey chow. Rhesus macaques are seasonal breeders. In the Cayo Santiago population, there is a 6-month mating season beginning in March, followed by a 6-month birth season beginning in September (Hoffman et al., 2008; Rawlins \& Kessler, 1986). During the nonbreeding season, ovarian steroid hormones are at stable low levels (Wilson, Gordon, \& Collins, 1982).

Between January and February 2008, 32 adult females were captured. Subjects were classified as high- $(n=10)$, middle- $(n=$ $11)$, or low- $(n=11)$ ranking on the basis of aggressive and submissive interactions collected in previous studies (Hoffman, Ayala, Mas-Rivera, \& Maestripieri, in press; Maestripieri, Hoffman, Anderson, Carter, \& Higley, 2009; Maestripieri, Hoffman, Fulks, \& Gerald, 2008). All adult females were multiparous. Their ages ranged from 7 to 26 years (mean \pm SEM $=15.64 \pm 0.97$ ) and parity status ranged from 2 to 18 offspring (mean \pm SEM $=$ $9.47 \pm 0.84)$. Twenty adult females were currently lactating and had a live infant (infant age range: 1-134 days; mean \pm SEM = $81.35 \pm 7.82 ; N=7$ female infants and $N=13$ male infants). Eleven adult females were currently not lactating and had no infant. One adult female had given birth to a live infant during the present breeding season but the infant subsequently died prior to CSF and blood sample collection. This adult female was excluded from further analyses due to the uniqueness of her reproductive status.

Lactating and nonlactating adult females did not differ significantly by age (lactating: $14.62 \pm 1.23$ years; nonlactating: $17.36 \pm$ 1.65 years; $t=1.33, d f=29$, NS), dominance rank (lactating: high $=6$; middle $=6$; low $=8$; nonlactating: high $=3$; middle $=$ 5 ; low $=3$; chi square: $0.82, d f=2$; NS), parity status (lactating: $9.45 \pm 1.15$ total infants; nonlactating: $9.55 \pm 1.30$ total infants; $t=0.05, d f=29$; NS), or any variables related to sampling (time of day, time since beginning of the procedure, and time since ketamine injection). All females were inspected by a veterinarian at the time of sampling and found to be in general good health. There was therefore no indication that currently nonlactating females in this study did not conceive as a result of health problems, or that currently lactating females were in poor health. All procedures were carried out in accordance with the National Institutes of Health Guide for the Care and Use of Laboratory Animals.

\section{Sampling Procedures and Hormone Assays}

Monkeys were captured in a feeding corral, approximately 100 $\mathrm{m}^{2}$, which was provisioned daily with monkey chow. Trapping generally occurred between 0830 and $1200 \mathrm{~h}$. Subjects were netted or captured by hand in the corral, transferred to a holding cage $(0.62 \times 0.42 \times 0.62 \mathrm{~m})$, and moved to a small field laboratory. The adult females and their infants (if they had one) were then placed into a standard squeeze cage for overnight housing. The following morning, blood and CSF samples were collected as described below. It is important to note that plasma cortisol and CSF oxytocin levels measured the morning after capture presumably reflect the cumulative stressful effects of capture and overnight housing (Hoffman et al. in press; Laudenslager et al., 1999; Maestripieri et al., 2008), rather than basal hormone levels in free-ranging monkeys.

Blood and CSF samples were collected from all adult females, and from 17 of the 20 infants $(N=5$ females, $N=12$ males $)$ Three infants $(N=2$ females and 1 male) were excluded from the study because they were either too young to sample safely or provided insufficient sample volumes. Anesthesia was induced using a sterile single-use syringe containing ketamine (approximately $10 \mathrm{mg} / \mathrm{kg}$ ), which was administered into the vastus lateralis muscle. Mother-infant pairs were separated only for the period of time when mothers were anesthetized. Blood samples were collected between 0715 and $1040 \mathrm{~h}$ (average time of day: 8:18 \pm 5.0 $\mathrm{min})$. Blood samples were taken, on average, $66.5 \pm 5.5 \mathrm{~min}$ after the door of the laboratory was first opened (range: 7-213 $\mathrm{min}$ ), and $22.1 \pm 2.7 \mathrm{~min}$ after ketamine administration (range 0-127 min). For each subject, CSF sample collection was initiated immediately after blood collection, and CSF sample collection was typically completed within 5 minutes of initiation. Time of sample collec- 
tion was not significantly correlated with CSF oxytocin levels $\left(r_{\mathrm{s}}=0.190 ; d f=46 ; \mathrm{p}=\mathrm{NS}\right)$ or plasma cortisol levels $\left(r_{\mathrm{s}}=\right.$ $-0.211 ; d f=46 ; \mathrm{p}=\mathrm{NS})$.

Blood samples (3-5 $\mathrm{ml}$ for adult females and 1-2 ml for infants) were collected from the femoral vein into heparin-treated vacutainer tubes. Samples were refrigerated for 20 minutes and were then centrifuged for 20 minutes. Plasma was aliquoted into microcentrifuge tubes that were stored at $-80{ }^{\circ} \mathrm{C}$ until shipped to the Biomarker Assay Core Lab of the Yerkes National Primate Research Center, where they were assayed in duplicate for cortisol by radioimmunoassay using a commercially available kit (Diagnostic Systems Laboratories, Webster, TX). Intra- and interassay coefficients of variation were below $15 \%$ and assay sensitivity is 0.50 $\mu \mathrm{g} / \mathrm{dl}$. Adult female plasma cortisol values examined in the present study are part of a larger study of stress and reproduction in multiparous females (Hoffman et al. in press). Infant plasma cortisol and all CSF oxytocin values reported herein are not part of this larger study.

CSF samples (approximately 1-2 $\mathrm{ml}$ for adult females and $1 \mathrm{ml}$ for infants) were collected from the cisterna magna using a sterile single-use needle. CSF was allowed to passively drip into a microcentrifuge tube and was subsequently flash-frozen on dry ice. The CSF samples were stored at $-80 \mathrm{C}$ until shipped to Dr. Parker's Neuroendocrinology laboratory at Stanford University. Unextracted CSF samples were then directly assayed in duplicate (100 $\mu \mathrm{l}$ per well) for oxytocin by enzyme immunoassay using a commercially available kit (Assay Designs Inc., Ann Arbor, MI). Peptide extraction is commonly used as a means by which to remove matrix interference effects from large proteins. The total protein in plasma is far greater and more complex than that in CSF (Fishman, 1992). Previous rhesus monkey research has used unextracted CSF samples to demonstrate nursery-reared monkeys exhibit diminished CSF oxytocin levels compared to maternally reared monkeys (Winslow et al., 2003). On the basis of this combined evidence we assayed unextracted CSF samples in this study. Intra- and interassay coefficients of variation were below $10 \%$ and assay sensitivity is $11.7 \mathrm{pg} / \mathrm{ml}$. Technicians were blind to experimental conditions while conducting all hormone assays.

\section{Statistical Analyses}

Mann-Whitney $(U)$ and Kruskal-Wallis $(\mathrm{H})$ tests were used to assess group differences in CSF oxytocin levels. These nonparametric tests were used because data were not normally distributed. Relationships between continuous variables were assessed with Spearman rank order correlations $\left(r_{\mathrm{s}}\right)$. Because individual differences in one measure can be associated with differences in another measure without the relationship being linear, rank order correlation statistics were more appropriate for these analyses than Pearson product-moment correlations. Test statistics were evaluated with two-tail probabilities $(\alpha<.05)$ and descriptive statistics are presented as mean \pm S.E.M.

\section{Results}

CSF oxytocin concentrations ranged from 36.02 to $134.41 \mathrm{pg} / \mathrm{ml}$ in adult females, and 35.94 to $77.3 \mathrm{pg} / \mathrm{ml}$ in infants. CSF oxytocin concentrations did not differ significantly between lactating and nonlactating adult females (lactating $=68.32 \pm 4.03 \mathrm{pg} / \mathrm{ml}$; non- lactating $=63.29 \pm 5.17 \mathrm{pg} / \mathrm{ml}$; Mann-Whitney test: $U=84$ $d f=1$; ns), and age of infant was not associated with CSF oxytocin concentrations in the lactating adult females $\left(r_{\mathrm{s}}=\right.$ $-0.276 ; d f=18$; ns). Although CSF oxytocin levels were lower in male $(54.29 \pm 4.02 \mathrm{pg} / \mathrm{ml})$ compared to female $(65.79 \pm 3.15$ $\mathrm{pg} / \mathrm{ml}$ ) infants, this trend did not reach statistical significance (Mann-Whitney test: $U=46 ; d f=1 ; p=.092$ ). Finally CSF oxytocin levels did not differ in relation to dominance rank for adult females (high: $65.14 \pm 3.63 \mathrm{pg} / \mathrm{ml}$; middle: $73.84 \pm 7.43$ pg/ml; low: $60.38 \pm 3.38 \mathrm{pg} / \mathrm{ml}$; Kruskal-Wallis test: $\mathrm{H}=1.35$; $d f=2 ; \mathrm{ns}$ ) or infants (high: $48.91 \pm 6.79 \mathrm{pg} / \mathrm{ml}$; middle: $61.49 \pm$ $3.40 \mathrm{pg} / \mathrm{ml}$; low: $61.16 \pm 5.73 \mathrm{pg} / \mathrm{ml}$; Kruskal-Wallis test: $\mathrm{H}=$ $2.53 ; d f=2 ; \mathrm{ns})$.

CSF oxytocin concentrations were significantly positively correlated with adult female age $\left(r_{\mathrm{s}}=0.367 ; d f=29 ; p=.04\right)$, such that older adult females had higher CSF oxytocin levels than younger adult females. When lactating and nonlactating adult female groups were considered separately, the correlation between CSF oxytocin levels and age was most evident in lactating $\left(r_{\mathrm{s}}=\right.$ $0.677 ; d f=18 ; p=.003)$ rather than nonlactating $\left(r_{\mathrm{s}}=0.109\right.$; $d f=9 ; \mathrm{p}=n s$ ), adult females (see Figure 1). In contrast, CSF oxytocin concentrations were significantly negatively correlated with infant age $\left(r_{\mathrm{s}}=-0.66 ; d f=15 ; p=.007\right)$, such that older infants had lower CSF oxytocin levels than younger infants (see Figure 1). Correlations between age and CSF oxytocin levels could not be considered separately for male and female infants due to the small sample size. No relationships between age and plasma cortisol levels were observed in any group.

Although adult females had higher CSF oxytocin concentrations compared to infants (adult females $=66.54 \pm 3.16 \mathrm{vs}$. infants $=$ $57.67 \pm 3.21 \mathrm{pg} / \mathrm{ml}$ ) this trend did not reach statistical significance (Mann-Whitney test: $U=195, d f=1, p=.14$ ). There was no significant correlation between CSF concentrations of oxytocin in mothers with those of their infants $\left(r_{\mathrm{s}}=0.37\right.$; $d f=15$; ns $)$, or between plasma concentrations of cortisol in mothers with those of their infants $\left(r_{\mathrm{s}}=0.045 ; d f=15 ; \mathrm{ns}\right)$. CSF oxytocin levels were not correlated with plasma cortisol levels in adult females when considered as a group $\left(r_{\mathrm{s}}=0.221 ; d f=29 ; p=n s\right)$ or when examined separately by reproductive status (lactating females: $r_{\mathrm{s}}=0.269 ; d f=18 ;$ ns; nonlactating females: $r_{\mathrm{s}}=-0.009 ; d f=$ 9; ns). No relationships between CSF oxytocin and plasma cortisol levels were discerned in infants when considered as a group $\left(r_{\mathrm{s}}=\right.$ 0.235; $d f=15$; ns). Correlations between CSF oxytocin and plasma cortisol levels could not be considered separately for male and female infants due to the small sample size.

\section{Discussion}

Our study demonstrates that CSF oxytocin levels can be reliably measured in free-ranging rhesus monkeys living in a naturalistic environment, as well as in infants as young as 1 month of age. The procedures used in our study and our preliminary data therefore can provide the basis for future work investigating the relationships between CSF oxytocin biology and social processes in socially and ecologically valid contexts.

Among adult female rhesus macaques, CSF oxytocin levels increased significantly with age, and this correlation was most pronounced in lactating females. Whether or not this observed difference is a function of statistical power (i.e., 20 lactating vs. 11 

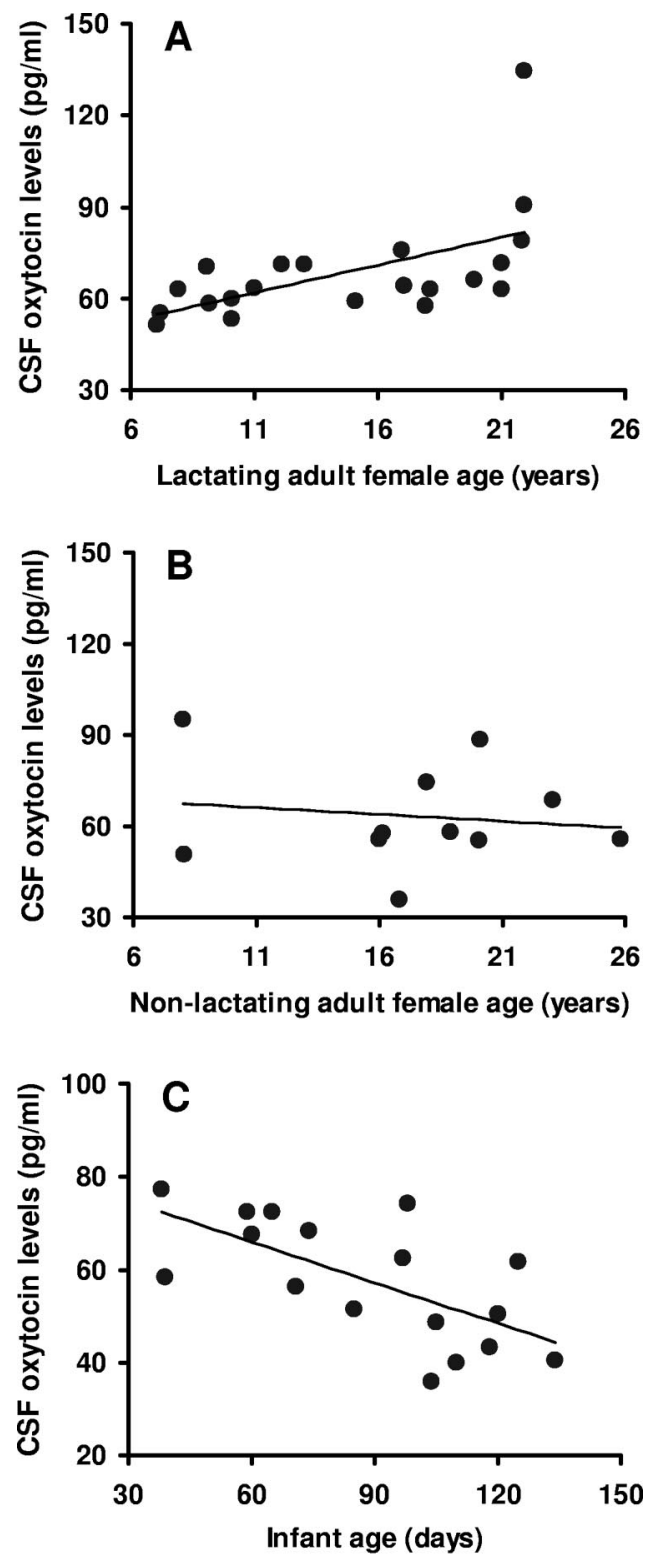

Figure 1. CSF oxytocin concentrations vary as a function of age in free-ranging rhesus macaques. Correlations between age and CSF oxytocin concentrations are presented for 20 adult lactating females (panel A), 11 nonlactating adult females (panel B), and 17 infants (panel C).

nonlactating adult females) or reflects a biologically significant effect merits further examination. In this rhesus population, the median life span for females that survive to reproductive age (4 years) is approximately 15 years, and the maximum life span is approximately 31 years (Hoffman, Higham, Mas-Rivera, Ayala, \& Maestripieri, under review). In this study, aging adult females (15-26 years) had generally higher CSF oxytocin levels than younger adult females (7-15 years). A similar finding has been reported in a study of humans, which compared plasma oxytocin levels in young and old men (22-40 vs. 60-75-year-old) (Forsling, Montgomery, Halpin, Windle, \& Treacher, 1998). Although the functional significance of aging-related increases in CSF oxytocin levels remains unknown, these findings suggest that CSF oxytocin levels may be used as a biomarker of aging in nonhuman and human primates, and may provide useful information about interindividual variation in the aging process.

The CSF oxytocin levels of young infants (between 38 and 134 days) were generally lower than those of adult females and not significantly correlated with those of their mothers. In contrast to what we found in adults, there was a strong negative correlation between CSF oxytocin and age in infants. No comparable data are available for human infants or monkey infants as young as those assessed in our study, but CSF concentrations of oxytocin were found to be stable in captive mother-reared rhesus monkeys repeatedly assessed at $18(\sim 27 \pm 3.5 \mathrm{pg} / \mathrm{ml}), 24(\sim 28 \pm 3 \mathrm{pg} / \mathrm{ml})$, and $36(\sim 27 \pm 2 \mathrm{pg} / \mathrm{ml})$ months of age (Winslow et al., 2003). Similar to the relationship detected between oxytocin and adult aging, the causes and functional significance of age-related changes in CSF oxytocin early in life need to be addressed by future studies.

In a previous study of this subject population, plasma concentrations of oxytocin were not correlated with age and were found to be higher in lactating $(311.41 \pm 15.32 \mathrm{pg} / \mathrm{ml})$ compared to nonlactating $(267.86 \pm 18.29 \mathrm{pg} / \mathrm{ml})$ females using identical blood sampling procedures as those used in the present study (Maestripieri et al., 2009). In the present study, however, we found no differences in CSF oxytocin concentrations between lactating and nonlactating females. Research involving captive rhesus monkeys has demonstrated that variations in the concentrations of oxytocin in CSF are independent of suckling stimuli and plasma oxytocin concentrations (Amico et al., 1990), which supports the notion that central and peripheral oxytocin systems may be functionally independent, at least with regard to nursing. Although plasma oxytocin samples were not available for analysis in the present study, follow up studies are required to examine the relationships between concomitantly collected CSF and plasma oxytocin samples from free-ranging monkeys in the context of different types of nonreproductive social behavior.

There is increasing interest in oxytocin biology and its relationships with psychosocial stress exposure, hormones of the hypothalamic-pituitary-adrenal (HPA) axis, and stress coping. Oxytocin is released into the brain and periphery in response to acute psychosocial stressors (Landgraf \& Neumann, 2004; Onaka, 2004), where it acts to attenuate stress-induced HPA axis activation in rats and monkeys (Parker, Buckmaster, Schatzberg, \& Lyons, 2005; Windle et al., 2004). In the aftermath of chronic psychosocial stress, however, CSF oxytocin levels are diminished in monkeys and humans (Heim et al., 2009; Winslow et al., 2003). In rhesus monkeys and other primates, low dominance rank is associated with chronic psychosocial stress and hyperactivation of the HPA axis (Sapolsky, 2005). Among female rhesus macaques on Cayo Santiago, low- and middle-ranking females exhibit higher cortisol responses to the stress of being captured and individually housed compared to high-ranking females (Maestripieri \& Hoffman, in press). In this study, we did not find significant effects of dominance rank on CSF oxytocin levels, or significant correlations between CSF oxytocin levels and plasma cortisol following the stress of overnight housing. A previous study of captive rhesus monkeys likewise detected no relationship between CSF oxytocin and plasma cortisol levels in monkeys exposed to early psychosocial adversity or control conditions (Winslow et al., 2003). In this 
latter study, monkeys were rapidly caught and sampled under controlled laboratory conditions, so the effects of capture stress on these neuroendocrine measures were minimized relative to those in our study. These collective findings highlight the fact that the interrelationships between plasma and CSF oxytocin biology, acute and chronic psychosocial stress exposure, plasma and CSF HPA axis hormones, and stress coping remain, at present, poorly understood.

As with all studies, ours is not without limitations. First, although CSF measurements of oxytocin are thought to reflect brain-released oxytocin, not all occurrences of brain-released oxytocin will be detectable in CSF measurements (Robinson \& Coombes, 1993). Therefore CSF oxytocin levels provide a gross measurement of central oxytocin activity, but do not permit detailed examination of the effects of regional oxytocin brain release (e.g., hypothalamic paraventricular nucleus) on dependent variables of interest (e.g., plasma cortisol). Second, the primary aim of this study was to determine the feasibility of measuring CSF oxytocin levels in free-ranging monkeys as a first step toward investigating the relationships between oxytocin biology and social functioning. To this end, biological samples were opportunistically collected during the annual trapping period which involves capture and overnight housing. It is therefore possible that a major factor influencing CSF oxytocin levels and plasma cortisol in this study was the high degree of stress associated with these procedures. The effects of stress, in turn, may have obscured relationships between baseline neuroendocrine values and the demographic variables examined in this study. Now that measurement feasibility has been established, follow-up studies are required to evaluate oxytocin biology under conditions which minimize the effects of capture stress on neuroendocrine values (i.e., under baseline conditions) and their relationships to social functioning. Third, the present study was not powered to examine the effects of infant gender on CSF oxytocin levels or to evaluate relationships between CSF oxytocin and plasma cortisol for male and female infants separately. Likewise, the present study did not consider the effects of nursing bouts or number of prior captures on the neuroendocrine measures, nor did it examine cortisol in CSF or oxytocin in plasma. Future studies should include examination of these variables which could further inform our understanding of oxytocin biology and social functioning in important ways.

In summary, these findings provide feasibility data for examining CSF oxytocin levels in rhesus monkeys under free-ranging conditions and in infants as young as 1 month of age. These data likewise add to the emerging and complicated picture of oxytocin biology across life span development (Carter, 2003; Forsling et al., 1998). Although the causes and functional significance of agerelated changes in CSF oxytocin early and late in life remain unclear, this peptide could represent a biomarker of central nervous system changes during early development and aging in human and nonhuman primates. Finally, concomitant measurement of CSF and plasma oxytocin levels, and assessments of their interrelationships with social behavior, psychological stress, and HPA axis physiology are required to more fully elucidate the distinct and shared contributions of central and peripheral oxytocin systems to social functioning and stress biology in free-ranging primates.

\section{References}

Amico, J. A., Challinor, S. M., \& Cameron, J. L. (1990). Pattern of oxytocin concentrations in the plasma and cerebrospinal fluid of lactating rhesus monkeys (Macaca mulatta): evidence for functionally independent oxytocinergic pathways in primates. Journal of Clinical Endocrinology and Metabolism, 71, 1531-1535.

Carter, C. S. (2003). Developmental consequences of oxytocin. Physiology and Behavior, 79, 383-397.

Carter, C. S. (2007). Sex differences in oxytocin and vasopressin: Implications for autism spectrum disorders? Behavioural Brain Research, 176, 170-186.

Carter, C. S., Grippo, A. J., Pournajafi-Nazarloo, H., Ruscio, M. G., \& Porges, S. W. (2008). Oxytocin, vasopressin and sociality. Progress in Brain Research, 170, 331-336.

Fishman, R. A. (1992). Cerebrospinal fluid in diseases of the nervous system. (2nd ed.). Philadelphia: Saunders.

Forsling, M. L., Montgomery, H., Halpin, D., Windle, R. J., \& Treacher, D. F. (1998). Daily patterns of secretion of neurohypophysial hormones in man: Effect of age. Experimental Physiology, 83, 409-418.

Gimpl, G., \& Fahrenholz, F. (2001). The oxytocin receptor system: Structure, function, and regulation. Physiological Reviews, 81, 629-683.

Heim, C., Young, L. J., Newport, D. J., Mletzko, T., Miller, A. H., \& Nemeroff, C. B. (2009). Lower CSF oxytocin concentrations in women with a history of childhood abuse. Molecular Psychiatry, 14, 954-958.

Hoffman, C. L., Ayala, J. E., Mas-Rivera, A., \& Maestripieri, D. (in press). Effects of reproductive condition and dominance rank on cortisol responsiveness to stress in free-ranging female rhesus macaques. American Journal of Primatology.

Hoffman, C. L., Higham, J. P., Mas-Rivera, A., Ayala, J. E., \& Maestripieri, D. (under review). Terminal investment and senescence in rhesus macaques (Macaca mulatta) on Cayo Santiago.

Hoffman, C. L., Ruiz-Lambides, A. V., Davila, E., Maldonado, E., Gerald, M. S., \& Maestripieri, D. (2008). Sex differences in survival costs of reproduction in a promiscuous primate. Behavioral Ecology and Sociobiology, 62, 1711-1718.

Landgraf, R., \& Neumann, I. D. (2004). Vasopressin and oxytocin release within the brain: A dynamic concept of multiple and variable modes of neuropeptide communication. Frontiers in Neuroendocrinology, 25, $150-176$.

Laudenslager, M. L., Rasmussen, K. L., Berman, C. M., Lilly, A. A., Shelton, S. E., Kalin, N. H., \& Suomi, S. J. (1999). A preliminary description of responses of free-ranging rhesus monkeys to brief capture experiences: Behavior, endocrine, immune, and health relationships. Brain, Behavior, and Immunity, 13, 124-137.

Lim, M. M., \& Young, L. J. (2006). Neuropeptidergic regulation of affiliative behavior and social bonding in animals. Hormones and Behavior, 50, 506-517.

Maestripieri, D., \& Hoffman, C. L. (in press). Behavior and social dynamics of rhesus macaques on Cayo Santiago. In Q. Wang (Ed.), Bones, genetics, and behavior: Physical anthropology at the Caribbean Primate Research Center. Berlin: Springer.

Maestripieri, D., Hoffman, C. L., Anderson, G. M., Carter, C. S., \& Higley, J. D. (2009). Mother-infant interactions in free-ranging rhesus macaques: Relationships between physiological and behavioral variables. Physiology and Behavior, 96, 613-619.

Maestripieri, D., Hoffman, C. L., Fulks, R., \& Gerald, M. S. (2008). Plasma cortisol responses to stress in lactating and nonlactating female rhesus macaques. Hormones and Behavior, 53, 170-176.

Modahl, C., Green, L., Fein, D., Morris, M., Waterhouse, L., Feinstein, C., \& Levin, H. (1998). Plasma oxytocin levels in autistic children. Biological Psychiatry, 43, 270-277.

Onaka, T. (2004). Neural pathways controlling central and peripheral oxytocin release during stress. Journal of Neuroendocrinology, 16, 308 312 . 
Parker, K. J., Buckmaster, C. L., Schatzberg, A. F., \& Lyons, D. M. (2005). Intranasal oxytocin administration attenuates the ACTH stress response in monkeys. Psychoneuroendocrinology, 30, 924-929.

Parker, K. J., Kenna, H., Zeitzer, J. M., Keller, J., Blasey, C. M., Amico, J. A., \& Schatzberg, A. F. (in press). Preliminary evidence that plasma oxytocin levels are elevated in major depression. Psychiatry Research.

Pedersen, C. A. (2004). Biological aspects of social bonding and the roots of human violence. Annals of the New York Academy of Sciences, 1036, $106-127$.

Rawlins, R. G., \& Kessler, M. J. (1986). The Cayo Santiago macaques: History, behavior, and biology. Albany, NY: SUNY Press.

Robinson, I. C., \& Coombes, J. E. (1993). Neurohypophysial peptides in cerebrospinal fluid: An update. Annals of the New York Academy of Sciences, 689, 269-284.

Rosenblum, L. A., Smith, E. L., Altemus, M., Scharf, B. A., Owens, M. J., Nemeroff, C. B., ... Coplan, J. D. (2002). Differing concentrations of corticotropin-releasing factor and oxytocin in the cerebrospinal fluid of bonnet and pigtail macaques. Psychoneuroendocrinology, 27, 651-660.
Sapolsky, R. M. (2005). The influence of social hierarchy on primate health. Science, 308, 648-652.

Wilson, M. E., Gordon, T. P., \& Collins, D. C. (1982). Variation in ovarian steroids associated with the annual mating period in female rhesus monkeys (Macaca mulatta). Biology of Reproduction, 27, 530-539.

Windle, R. J., Kershaw, Y. M., Shanks, N., Wood, S. A., Lightman, S. L., \& Ingram, C. D. (2004). Oxytocin attenuates stress-induced c-fos mRNA expression in specific forebrain regions associated with modulation of hypothalamo-pituitary-adrenal activity. Journal of Neuroscience, 24, 2974-2982.

Winslow, J. T., Noble, P. L., Lyons, C. K., Sterk, S. M., \& Insel, T. R. (2003). Rearing effects on cerebrospinal fluid oxytocin concentration and social buffering in rhesus monkeys. Neuropsychopharmacology, 28, 910-918.

Received January 14, 2010

Revision received March 19, 2010

Accepted March 19, 2010 